\title{
DÉFORMATION LOCALISÉE DE SURFACES DE RIEMANN
}

\author{
PETER HAÏSSINSKY
}

Abstract

Let $Y$ be a Riemann surface with compact boundary embedded into a hyperbolic Riemann surface of finite type $X$. It is proved that the space of deformations $\mathcal{D}$ of $Y$ into $X$ is an open subset of the Teichmüller space $\mathcal{T}(X)$ of $X$. Furthermore, $\mathcal{D}$ has compact closure if and only if $Y$ is simply connected or isomorphic to a punctured disk, and $\mathcal{D}=\mathcal{T}(X)$ if and only if the components of $X \backslash Y$ are all disks or punctured disks.

Soit $X$ une surface de Riemann hyperbolique de type fini. Changer sa structure complexe peut apparaître comme un phénomène purement local, alors que sa métrique hyperbolique est perturbée globalement. Dans ce travail, nous tâchons d'expliquer l'influence du changement de structure complexe sur la structure hyperbolique.

A cette fin, on considère $Y \subset X$ une surface connexe fermée à bord $\partial Y \cap X$ compact. Le résultat principal est alors le suivant.

\section{Théorème.}

(i) L'espace des déformations $\mathcal{D}$ de la structure complexe de $Y$ dans $X$ forme un ouvert de l'espace de Teichmüller de X.

(ii) L'espace $\mathcal{D}$ est relativement compact si et seulement si l'injection de $Y$ dans $X$ est homotope à un point ou à un disque épointé.

(iii) L'espace $\mathcal{D}$ recouvre tout l'espace de Teichmüller de $X$ si et seulement si chaque composante connexe de $X \backslash Y$ est un disque éventuellement épointé.

L'énoncé du point (i) est dû indépendamment à J.-P. Demailly et à C. T. McMullen. Le point (ii) contredit une rumeur qui affirmait au contraire que l'on pouvait atteindre tous les points de l'espace de Teichmüller en ne changeant la structure complexe de la surface initiale

2000 Mathematics Subject Classification. 30F60, 32G15.

Key words. Teichmüller theory, hyperbolic geometry, simple closed curves. 
que sur un disque (voir aussi la Proposition 3.1). Le point (iii) indique la taille minimale de la sous-surface nécessaire pour effectivement couvrir tout l'espace.

On applique ce théorème à la variation de Schiffer.

Corollaire. Les déformations d'une surface de Riemann hyperbolique de type fini induites par la variation de Schiffer restent dans un compact de l'espace de Teichmüller.

Démonstration: La variation de Schiffer consiste à inciser la surface le long d'un arc plongé et d'y recoller un disque. Cette déformation peut être décrite par une déformation de $X$ localisée dans un voisinage de l'arc [4]. Par conséquent, le point (ii) s'applique.

Dans le dernier paragraphe, on précise le point (ii) du théorème en montrant que plus le disque est petit, plus les déformations possibles sont restreintes. Ceci répond à une question de F. Loray.

Remerciements. Ce travail est issu de discussions informelles que j'ai eues ces dernières années sur des déformations restreintes à des disques. Je tiens plus particulièrement à remercier J. H. Hubbard et C. T. McMullen qui ont eu la bonté de partager leurs visions concrètes de l'espace de Teichmüller. J'aimerais aussi remercier F. Loray pour l'intérêt qu'il a porté sur ce travail.

\section{Une construction de l'espace de Teichmüller de $X$}

Cette présentation s'inspire de l'ouvrage [4]. Nous prions le lecteur intéressé de s'y reporter pour obtenir les justifications nécessaires des affirmations qui suivent. En ce qui concerne la théorie des homéomorphismes quasiconformes, l'ouvrage [2] contient les démonstrations des résultats utilisés ici.

On considère une surface de Riemann $X$ quelconque. Soit $\mathcal{B}(X)$ l'espace vectoriel des formes de Beltrami sur $X$ i.e., l'espace des $(-1,1)$-formes différentielles mesurables essentiellement bornées sur $X$. Muni de la norme du sup $\|\cdot\|_{\infty}$, cet espace est un espace de Banach sur $\mathbb{C}$. On note $\operatorname{Def}(X)$ la boule unité de $\mathcal{B}(X)$. Se donner $\mu \in \operatorname{Def}(X)$ revient par le théorème de Riemann mesurable à se donner un homéomorphisme quasiconforme $\varphi: X \rightarrow Z$, où $Z$ est une autre surface de Riemann définie à application conforme près et où $\varphi$ vérifie au sens des distributions l'équation dite de Beltrami

$$
\frac{\partial \varphi}{\partial \bar{z}}=\mu \cdot \frac{\partial \varphi}{\partial z}
$$


L'espace $Q C_{0}(X)$ des homéomorphismes quasiconformes $\omega: X \rightarrow X$ isotopes à l'identité agit $\operatorname{sur} \operatorname{Def}(X) \operatorname{par} \omega \cdot(\varphi, Z)=\left(\varphi \circ \omega^{-1}, Z\right)$. L'espace quotient $\operatorname{Def}(X) / Q C_{0}(X)$ est l'espace de Teichmüller de $X$ que l'on notera $\mathcal{T}(X)$.

Lorsque $X$ est de type fini (surface de Riemann compacte avec un nombre au plus fini de point(s) enlevé(s)), cet espace est muni d'une structure de variété complexe de dimension finie telle que la projection canonique $\varpi: \operatorname{Def}(X) \rightarrow \mathcal{T}(X)$ est holomorphe. L'espace cotangent de $\mathcal{T}(X)$ en un point $([\varphi], Z)$ s'identifie à l'espace $Q(Z)$ des formes différentielles quadratiques holomorphes intégrables via l'accouplement

$$
\langle\mu, q\rangle=\int_{Z} \mu \cdot q .
$$

L'espace tangent s'identifie alors à $\mathcal{B}(Z) / N_{Z}$, où

$$
N_{Z}=\{\mu \in \mathcal{B}(Z),\langle\mu, q\rangle=0 \forall q \in Q(Z)\} .
$$

Dans le contexte de notre théorème, on définit l'application $\Phi$ : $\operatorname{Def}(Y) \rightarrow \operatorname{Def}(X)$ par $\left.\Phi(\mu)\right|_{Y}=\mu$ et $\left.\Phi(\mu)\right|_{X \backslash Y}=0$. On pose $\phi=$ $\varpi \circ \Phi: \operatorname{Def}(Y) \rightarrow \mathcal{T}(X)$. Cette application est holomorphe et on note $\mathcal{D}=\phi(\operatorname{Def}(Y))$. Le premier point du théorème revient à montrer la proposition suivante.

Proposition 1.1. Si $X$ est de type fini, alors pour tout $\mu \in \operatorname{Def}(Y)$, l'application linéaire tangente $D_{\mu} \phi$ est surjective.

Démonstration: Traitons d'abord le cas de la surjectivité au point $\mu=0$. Si $D \phi$ n'est pas surjective, alors il existe $q \in Q(X)$ telle que, pour tout $\mu \in \operatorname{Def}(Y)$, on ait $\langle\Phi(\mu), q\rangle=0$. Pour $q \in Q(X)$, on définit

$$
\mu_{q}=\frac{\bar{q}}{|q|} \cdot \chi_{Y}
$$

où $\chi_{Y}$ désigne la fonction indicatrice de $Y$. Il s'ensuit que $\mu_{q}$ est un élément de $\mathcal{B}(Y)$ et

$$
\left\langle\Phi\left(\mu_{q}\right), q\right\rangle=\int_{Y} \frac{\bar{q}}{|q|} \cdot q=\int_{Y}|q|>0 .
$$

Ceci établit la surjectivité de $D \phi$ à l'identité.

Etudions maintenant l'application linéaire tangente en un point $\mu \epsilon$ $\operatorname{Def}(Y)$. On se ramène à l'argument précédent de la manière suivante. Notons $\Phi(\mu)=\left(f, X^{\prime}\right)$ et $Y^{\prime}=f(Y)$. On considère les applications analogues $\Phi^{\prime}: \operatorname{Def}\left(Y^{\prime}\right) \rightarrow \operatorname{Def}\left(X^{\prime}\right), \varpi^{\prime}: \operatorname{Def}\left(X^{\prime}\right) \rightarrow \mathcal{T}\left(X^{\prime}\right)$, ainsi que $\phi^{\prime}: \operatorname{Def}\left(Y^{\prime}\right) \rightarrow \mathcal{T}\left(X^{\prime}\right)$. La précomposition par $f^{-1}$ induit des isomorphismes $\chi: \operatorname{Def}(Y) \rightarrow \operatorname{Def}\left(Y^{\prime}\right), \Psi: \operatorname{Def}(X) \rightarrow \operatorname{Def}\left(X^{\prime}\right)$ et $\psi: \mathcal{T}(X) \rightarrow$ 
$\mathcal{T}\left(X^{\prime}\right)$ tels que $\chi(\mu)=0$ et $\Psi(\Phi(\mu))=\left(\mathrm{id}, X^{\prime}\right)$, et tels que $\phi=\psi^{-1}$ 。 $\phi^{\prime} \circ \chi$.

Par conséquent, la surjectivité de $D \phi$ au point $\mu$ est équivalente à celle de $D \phi^{\prime}$ à l'origine, qui découle du même argument que ci-dessus.

\section{Fonctions longueurs}

Soit $\gamma$ une courbe fermée simple de $X$. On considère l'application $\ell_{\gamma}: \mathcal{T}(X) \rightarrow \mathbb{R}_{+}$qui associe à tout point $([\varphi], Z) \in \mathcal{T}(X)$ la longueur hyperbolique de la géodésique sur $Z$ isotope à $\varphi(\gamma)$. Cette application est toujours continue (voir $[\mathbf{1}],[\mathbf{3}]$ ).

Le reste de la démonstration du théorème s'appuie sur la proposition suivante.

Proposition 2.1. Soit $\gamma$ une courbe fermée simple de $X \backslash Y$. Si $\ell_{\gamma}([\mathrm{id}], X)>0$ alors il existe une constante (finie) $C>0$ telle que $\ell_{\gamma}(\phi(\mu)) \leq C$ pour tout $\mu \in \operatorname{Def}(Y)$.

Démonstration: Puisque $\gamma \cap Y=\emptyset$, il existe un anneau plongé $A(\gamma) \subset$ $X \backslash Y$ d'équateur $\gamma$. Il s'ensuit que

$$
0<\bmod A(\gamma)=\frac{\pi}{\ell_{A(\gamma)}(\gamma)}
$$

où $\ell_{A(\gamma)}$ désigne la longueur hyperbolique dans la surface $A(\gamma)$. Comme l'inclusion contracte la métrique hyperbolique, on a aussi $\ell_{X}(\gamma) \leq \ell_{A(\gamma)}(\gamma)$.

Soit $\mu \in \operatorname{Def}(Y)$, on note $\Phi(\mu)=(\varphi, Z)$. Alors $\left.\varphi\right|_{A(\gamma)}$ est conforme donc $\bmod A(\gamma)=\bmod \varphi A(\gamma)$. Du coup,

$$
\ell_{\gamma}(\phi(\mu)) \leq \ell_{Z}(\varphi(\gamma)) \leq \ell_{\varphi A(\gamma)}(\varphi(\gamma))=\ell_{A(\gamma)}(\gamma)
$$

Suivant S. P. Kerckhoff [5], on dit qu'une famille de courbes fermées simples $\Gamma$ de $X$ remplit $X$ si les courbes sont dans des classes d'isotopies distinctes, si $\ell_{\gamma}([\mathrm{id}])>0$ pour tout $\gamma \in \Gamma$, et si chaque composante de $X \backslash\left(\cup_{\Gamma} \gamma\right)$ est un disque éventuellement épointé.

On reconnaît la compacité relative d'un sous-ensemble de $\mathcal{T}(X)$ grâce à la proposition suivante.

Proposition 2.2. Soit $X$ une surface de type fini. Si $\Gamma$ remplit $X$ alors l'application

$$
\ell_{\Gamma}: \mathcal{T}(X) \rightarrow \mathbb{R}_{+} \backslash\{0\}
$$

définie $\operatorname{par} \ell_{\Gamma}(\tau)=\sum_{\gamma \in \Gamma} \ell_{\gamma}(\tau)$ est propre. 
Démonstration: ( $c f$. Lemme 3.1 de [5]). Soit $\widehat{\gamma}$ une courbe fermée simple de $X$ telle que $\ell_{\widehat{\gamma}}([\mathrm{id}])>0$. Montrons que $\log \ell_{\widehat{\gamma}}$ reste bornée quand on parcourt l'ensemble

$$
\mathcal{B}_{K}=\left\{\tau \in \mathcal{T}(X), \ell_{\Gamma}(\tau) \leq K\right\},
$$

pour un $K>0$ assez grand.

Il existe $N=N(\widehat{\gamma})$ telle que $\widehat{\gamma}$ soit homotope à une courbe incluse dans $\cup_{\gamma \in \Gamma} \gamma$ de manière à ce que chaque point de $\cup_{\gamma \in \Gamma} \gamma$ n'est pas parcouru plus de $N$ fois. Du coup, $\ell_{\widehat{\gamma}}(\tau) \leq N \cdot \ell_{\Gamma}(\tau)$ pour tout $\tau \in \mathcal{T}(X)$.

D'autre part, puisque $\Gamma$ remplit $X, \widehat{\gamma}$ rencontre au moins une courbe $\gamma \in \Gamma$. Si $\ell_{\widehat{\gamma}}$ pouvait tendre vers zéro, alors la longueur $\ell_{\gamma}$ devrait tendre vers l'infini par le lemme du col. Ceci établit l'affirmation.

On considère maintenant une famille $\widehat{\Gamma}$ de géodésiques fermées simples de $X$ deux à deux disjointes maximale. Elle nous permet de paramétrer $\mathcal{T}(X)$ par les coordonnées de Fenchel-Nielsen [1]. Chaque courbe $\widehat{\gamma} \in \widehat{\Gamma}$ induit deux paramètres, l'un est $\log \ell_{\widehat{\gamma}}$, l'autre est un paramètre d'enroulement : on peut découper $X$ le long de $\widehat{\gamma}$ et recoller ses deux nouvelles extrémités isométriquement en effectuant un décalage de $\theta \in \mathbb{R}$ tours; ce nombre $\theta$ mesure l'enroulement.

On reste dans un compact de $\mathcal{T}(X)$ si ces paramètres restent dans un compact de $\mathbb{R}^{\widehat{\Gamma}}$. D'après l'argument ci-dessus, on sait déjà que $\log \ell_{\widehat{\gamma}}$ reste borné pour toute courbe $\widehat{\gamma} \in \widehat{\Gamma}$. Reste à comprendre l'effet de l'enroulement d'une courbe $\widehat{\gamma} \in \widehat{\Gamma}$ sur une courbe $\gamma \in \Gamma$ qui rencontre $\widehat{\gamma}$. Lorsque l'enroulement de $\widehat{\gamma}$ augmente, la courbe $\gamma$ s'enroule d'autant (ou presque) autour de $\hat{\gamma}$, donc sa longueur est au moins l'enroulement de fois $\ell_{\widehat{\gamma}}$. Donc cet enroulement doit rester borné.

Par conséquent, l'ensemble $\mathcal{B}_{K}$ est borné donc compact dans $\mathcal{T}(X)$, puisque c'est un espace de dimension finie.

\section{Démonstration du théorème:}

(1) S'il existe une courbe $\gamma$ fermée simple disjointe de $Y$ telle que $\ell_{\gamma}([$ id] $])>0$, alors la Proposition 2.1 montre que $\phi$ ne peut être surjective.

(2) En particulier, si $Y$ est homotope à un point ou à un disque épointé, alors il existe une famille de courbes $\Gamma$ disjointes de $Y$ qui remplit $X$. Avec la Proposition 2.2, on en déduit que $\mathcal{D}$ est relativement compacte dans $\mathcal{T}(X)$. Ceci établit la suffisance de (ii).

Réciproquement, s'il existe $\gamma \subset Y$ telle que $\ell_{\gamma}([\mathrm{id}])>0$, alors on peut trouver un anneau $A \subset Y$ d'équateur $\gamma$ et on peut déformer sa structure complexe pour que le module de cet anneau tende vers 
l'infini. Cela implique que l'image de $\operatorname{Def}(Y)$ ne sera pas bornée. La nécessité de (ii) est ainsi établie.

(3) Le point (1) montre la nécessité de (iii). Il reste donc à montrer que $\phi$ est surjective. On suppose donc que les composantes connexes de $X \backslash Y$ sont des disques éventuellement épointés. Soit $\tau=([\varphi], Z) \in \mathcal{T}(X)$. Notons $V_{1}, \ldots, V_{k}$ les composantes connexes de $X \backslash Y$.

Comme $Y$ est à bord compact, il existe des disques ouverts éventuellement épointés $W_{j}$, voisinages de $\overline{V_{j}}$, d'intérieurs deux à deux disjoints. Il suffit de montrer que $\left.\varphi\right|_{W_{j}}$ est isotope rel. $\partial W_{j}$ à un homéomorphisme $\psi_{j}$, conforme sur $V_{j}$. Pour cela, on peut se ramener à la situation où $W_{j}=\varphi\left(W_{j}\right)=\mathbb{D}, V_{j}=D(0, r)$ pour un certain $1>r>0$, et $\varphi\left(V_{j}\right)$ est un domaine de Jordan relativement compact à bord lisse de $\mathbb{D}$.

En utilisant le théorème de Riemann mesurable, on construit une isotopie $\left(\psi_{t}\right)$ de $\left.\varphi\right|_{D(0, r)}$ de $\psi_{0}=\varphi$ à une application conforme $\psi_{1}$ qui fixe 0 pour tout $t$ et qui préserve $\varphi(D(0, r))$. Ensuite, en passant aux revêtements universels de $\mathbb{D} \backslash \overline{D(0, r)}$ et de $\mathbb{D} \backslash \overline{\varphi D(0, r)}$, l'extension de Douady-Earle appliquée aux relevés de $\left.\varphi\right|_{\{|z|=1\}}$ et $\left.\psi_{t}\right|_{\{|z|=r\}}$ nous fournit l'isotopie recherchée [4].

On définit $\widehat{\varphi}: X \rightarrow Z$ par $\left.\widehat{\varphi}\right|_{X \backslash \cup W_{j}}=\varphi$, et $\left.\widehat{\varphi}\right|_{W_{j}}=\psi_{1}$. On a par construction $[\widehat{\varphi}]=[\varphi]$ donc $\tau \in \mathcal{D}$.

\section{Petites déformations}

L'objet de paragraphe est d'établir la proposition suivante.

Proposition 3.1. Soient $X$ une surface hyperbolique de type fini et $x \in X$; pour tout voisinage de $([\mathrm{id}], X)$ dans $\mathcal{T}(X)$, il existe $r_{0}>0$ telle que, pour tout $0<r<r_{0}$, les déformations restreintes au disque centré en $x$ et de rayon $r$ sont contenues dans ce voisinage.

Démonstration: Si $r>0$, on note $Y_{r}=\overline{D(x, r)}, X_{r}=X \backslash Y_{r}$, et $\Phi_{r}: \operatorname{Def}\left(Y_{r}\right) \rightarrow \operatorname{Def}(X)$ l'application définie ci-dessus. On note que si $r<r^{\prime}$ alors $\Phi_{r}\left(\operatorname{Def}\left(Y_{r}\right)\right) \subset \Phi_{r^{\prime}}\left(\operatorname{Def}\left(Y_{r^{\prime}}\right)\right)$.

Pour montrer la proposition, il suffit donc de montrer que $\cap_{r>0} \Phi_{r}\left(\operatorname{Def}\left(Y_{r}\right)\right)=\{([\mathrm{id}], X)\}$.

On se donne $(\varphi, Z) \in \cap_{r>0} \Phi_{r}\left(\operatorname{Def}\left(Y_{r}\right)\right)$ et montrons que $(\varphi, Z)=$ (id, $X)$. Par définition, il existe, pour chaque $r>0$ assez petit, une application holomorphe injective $\varphi_{r}: X_{r} \rightarrow Z$. Si on munit $X_{r}$ et $Z$ de 
la métrique hyperbolique, alors l'application $\varphi_{r}$ est 1-Lipschitzienne par le lemme de Schwarz-Pick, donc la famille $\left(\varphi_{r}\right)_{r}$ est équicontinue sur tout compact de $X \backslash\{x\}$.

$X$ étant de type fini, $\left(\varphi_{r}\right)$ préserve les épointements ainsi que les classes d'isotopie de courbes non homotopes à un point ou un épointement (dans $X$ et $Z$ ). Du coup, $\left(\varphi_{r}\right)$ est une famille normale, et toute limite est non constante. Ceci implique qu'il existe une application holomorphe injective $\psi: X \backslash\{x\} \rightarrow Z$. Or le point $x$ est une singularité effaçable, donc $\psi$ réalise un isomorphisme entre $X$ et $Z$.

\section{Références}

[1] W. AвікоғF, "The real analytic theory of Teichmüller space", Lecture Notes in Mathematics 820, Springer, Berlin, 1980.

[2] L. V. AhLFors, "Lectures on quasiconformal mappings", Van Nostrand Mathematical Studies 10, D. Van Nostrand Co., Inc., Toronto, Ont.-New York-London, 1966.

[3] A. Fathi, F. Laudenbach et V. PoÉnaru, Travaux de Thurston sur les surfaces, Astérisque 66-67 (1979).

[4] F. Gardiner, P. Frederick et N. Lakic, "Quasiconformal Teichmüller theory", Mathematical Surveys and Monographs 76, American Mathematical Society, Providence, RI, 2000.

[5] S. P. Kerckhoff, The Nielsen realization problem, Ann. of Math. (2) $\mathbf{1 1 7 ( 2 )}$ (1983), 235-265.

\section{LATP/CMI}

Université de Provence

39, rue Frédéric Joliot-Curie

13453 Marseille cedex 13

France

E-mail address: phaissin@cmi.univ-mrs.fr 